\title{
DESARROLLO DE HABILIDADES INFORMACIONALES (DHI) COMO COMPETENCIA TRANSVERSAL EN ESTUDIANTES DE LA PONTIFICIA UNIVERSIDAD JAVERIANA CALI: CURSO VIRTUAL DE BIBLIOTECA UNA PROPUESTA DE FORMACIÓN
}

\section{INFORMATIONAL SKILLS DEVELOPMENT (DHI) CROSS COMPETITION AS STUDENTS JAVERIANA UNIVERSITY: VIRTUAL LIBRARY A PROPOSED COURSE OF TRAINING}

\author{
Nancy Vanegas ${ }^{1}$, Andrea Serna ${ }^{1}$, María Fernanda Robles ${ }^{1}$, Tatiana Valencia ${ }^{1}$ \\ Recibido para publicación: 19 de mayo de 2014 - Aceptado para publicación: 15 julio de 2014
}

\begin{abstract}
RESUMEN
En el presente artículo se presenta la descripción y resultados del proyecto: Desarrollo de Habilidades Informacionales (DHI) como competencia transversal en estudiantes de la Pontificia Universidad Javeriana Cali, que consistió en la implementación en tres asignaturas presenciales de pregrado de los módulos virtuales que hacen parte del curso virtual de Biblioteca (dispuesto en la plataforma educativa Blackboard) ofrecido en el marco de su programa de formación de usuarios. Este estudio piloto aplicado en el periodo 2013 - 2014, se inscribe en una metodología mixta con carácter descriptivo y su propósito fue el de identificar los cambios en el acceso y uso de la información a partir de la implementación de los módulos del curso virtual de biblioteca. Los resultados obtenidos demuestran el aumento en el acceso y uso de los recursos de Biblioteca y una curva de aprendizaje ascendente que evidenciaba el fortalecimiento de habilidades informacionales comunes entre los objetivos de los módulos del curso virtual y los propuestos en la asignatura presencial.
\end{abstract}

Palabras clave: Alfabetización Informacional (ALFIN), desarrollo de habilidades informacionales (DHI), competencias, ambientes virtuales de aprendizaje (AVA).

\begin{abstract}
This article presents the description and result of the project: Informational Skills Development (IHD) as a transverse competence for the students in the Pontificia Universidad Javeriana Cali, which consisted of three classroom based courses in undergraduate programs. These courses were parts of the Virtual Course Library Set (provisions of the Blackboard learning platform) offered as part of its program of user training. This pilot study applied in the period 2013 - 2014, is part of a mixed and descriptive methodology and its purpose was to identify changes in access to and use of information from the implementation of online course modules from the library set. The results show an increase in access and use of library resources and learning curve upward evident strengthening common information skills among the objectives of the course modules and virtual classroom given subject.
\end{abstract}

Keywords: Information Literacy (ALFIN), informational skills, transversal competences, virtual learning environment (VLE).

${ }^{1}$ Pontificia Universidad Javeriana Cali 


\section{INTRODUCCIÓN}

Este artículo presenta los resultados del proyecto desarrollo de habilidades informacionales (DHI) como competencia transversal en estudiantes de la Pontificia Universidad Javeriana Cali, en un principio se describen las condiciones globales en donde cobra sentido la problemática del $\mathrm{DHI}$ desde un contexto global hacia el contexto local, en donde se hace evidente el papel que las universidades tienen en dicha formación especialmente a través de la Biblioteca.

A partir de lo anterior, en la siguiente sección se hace énfasis en la transformación de las Bibliotecas universitarias hacia un rol formativo que busca asumir y aportarle a la Educación Superior de acuerdo a las demandas que la sociedad de la información y del conocimiento le exige en relación con las necesidades de aprendizaje y desarrollo de competencias. En esta vía la cuarta sección describe cómo la Biblioteca de la universidad Javeriana le apuesta a esta transformación al pasar de una formación tradicional que privilegia la información sobre recursos y servicios a la formación basada en competencias informacionales apoyadas en las tecnologías de la información y comunicación (TIC) [1], a través de la estrategia de un curso virtual en el marco de su programa de formación de usuarios. La sección se expone la ruta metodológica con la que se desarrolla el estudio piloto del curso virtual integrado a 3 cursos presenciales 2 de expresión oral y escrita que corresponde al núcleo fundamental (comunicación oral y escrita, habilidades de la información para el desarrollo de producción) y uno de fundamentos de la Investigación que hace parte de la línea metodológica (aporte a la investigación). En la sección VI se muestran los resultados de la experiencia piloto del Curso "un viaje por el mundo del conocimiento" a partir de las categorías de análisis propuestas: datos sociodemográficos, apropiación del curso virtual y cambios en el acceso y uso de información (competencias informacionales) y finalmente la última sección donde se presentan las conclusiones.

\section{MARCO CONTEXTUAL}

La evolución que en los últimos años han tenido los modelos educativos en la enseñanza de la educación superior es directa consecuencia de los cambios que en nuestra sociedad están aconteciendo. UNESCO en su informe mundial del 2005 titulado: "Hacia las sociedades del conocimiento" describe este fenómeno de la siguiente manera: "...Los cambios radicales provocados por la tercera revolución industrial-la de las nuevas tecnologías han creado de hecho una nueva dinámica, porque desde mediados del siglo XX la formación de las personas y los grupos, así como los adelantos científicos y técnicos y las expresiones culturales, están en constante evolución, sobre todo hacia una interdependencia cada vez mayor." [2]

Este contexto ha llevado a que todos los países examinen la manera cómo acercan el mundo productivo con el educativo. Diferentes posiciones frente a esta demanda global se han presentado en el mundo, como la perspectiva del Espacio Europeo de Enseñanza Superior (EEES) que se centra en competencias transversales y otras como las lideradas por el Reino Unido y Australia que se han centrado en el desarrollo de competencias relacionadas con las ocupaciones mismas. Independientemente de cual sea el camino correcto es importante identificar cómo formar a los profesionales a la luz de las necesidades del mundo productivo de manera contextualizada. El pensar entonces en competencias transversales en Educación Superior está relacionado con la necesidad de ser países más competitivos frente a los retos de un mundo globalizado, disminuir la brecha existente entre lo profesional y lo laboral y aportar a la articulación entre educación superior y desarrollo social.

En este sentido el Ministerio de Educación Nacional (MEN) en Colombia, entre los años 2008 y 2009 identificó las principales competencias genéricas a desarrollar en Educación Superior 
tales como: a) comunicación en lengua materna y en otra lengua internacional, b) pensamiento matemático, c) ciudadanía, d) cultura científica y tecnológica y gestión de información, indispensables para el desempeño académico y laboral en Colombia. [3]

Con relación a la competencia genérica: cultura científica y tecnológica y gestión de información, en la última década las Bibliotecas Universitarias han tenido un interesante impulso. Evidencia de esto son los resultados encontrados en la investigación relacionada con la producción de materiales o recursos (artículos, ponencia, libros, trabajos de grado, tesis, modelos, eventos) en torno al tema de la Alfabetización informacional, realizada por el grupo de investigación "Información, Conocimiento y Sociedad" liderado por Alejandro Uribe Tirado de la Universidad de Antioquia. De las 337 universidades investigadas, solo 75 (el 22.3\%) de las IES evidenciaban en sus portales, información sobre formación de usuarios de las cuales 26 estaban en el nivel 1 y 37 en el nivel 2. De este grupo las que ya incluían en sus contenidos información desde la comprensión integral de la Alfabetización Informacional (ALFIN) eran 12, 8 en el nivel 1 y 4 en el nivel 2, de las cuales la Pontificia Universidad Javeriana - Cali se encontraba en el grupo de nivel 1.

Los autores describen en el Estado del arte del documento los niveles así: formación de Usuarios. Nivel 1: solo capacitación en los servicios generales de la biblioteca; formación de Usuarios. Nivel 2: capacitación en servicios generales de la biblioteca y algunos cursos -muy instrumentales- para búsqueda de información: utilización de catálogos/bases de datos. Alfabetización Informacional. Nivel 1: cursos desde la biblioteca para formar en competencias informacionales: lo instrumental, aprendizaje para toda la vida y pensamiento crítico. Alfabetización Informacional. Nivel 2: cursos desde la biblioteca para formar en competencias informacionales: incluye lo instrumental, aprendizaje para toda la vida, pensamiento crítico; y cursos/módulos específicos inmersos oficialmente en los currículos de distintos programas académicoscarreras para formar de manera transversal y disciplinar en esas competencias. [4]

A partir de este contexto, la Pontificia Universidad Javeriana de Cali interesada en desarrollar la competencia cultura científica y tecnológica y gestión de la información que consiste en "Comprender el mundo natural en que vivimos, aprender a transformarlo y manejar eficiente y responsablemente toda la información y el conocimiento que sobre él ha acumulado la humanidad a través de los milenios"[3] ha contado con el apoyo de la Biblioteca General que en alianza estratégica con la Oficina para el Fomento del Uso de las Tecnologías de Información y Comunicación - Javevirtual, propone el curso virtual "Un viaje por el mundo del conocimiento" en la plataforma institucional Blackboard, para formar y aportar al desarrollo de estas competencias de carácter transversal en sus estudiantes.

\section{LA BIBLIOTECA: DEL ROL INFORMATIVO Y DE SERVICIO AL ROL FORMATIVO}

Uno de los conceptos tradicionales sobre la Biblioteca Universitaria es el expresado por los autores Thompson y Carr, 1995: "La biblioteca es el corazón de la universidad, un motor para hacerla conservadora, transmisora y creadora de saber."[5]

Si bien la anterior postura fue asumida durante un tiempo, ahora el reto de la biblioteca es trascender esta postura y lanzarse a experimentar nuevas estrategias y servicios que impulsen la interactividad y la comunicación con los usuarios de una manera más potente y creativa. Para lograrlo, debe promover la formación de sus usuarios y desarrollar en ellos las habilidades informativas necesarias para hacer uso eficiente de los recursos de información, 
así mismo, formarlos en la adquisición de una serie de competencias, habilidades, destrezas y conocimientos que los dote de autonomía y pensamiento crítico frente a la actual sociedad de la información y del conocimiento.

En este sentido, la Biblioteca de la Pontificia Universidad Javeriana Cali deberá ser un centro de recursos y servicios de información que gestiona todos los fondos bibliográficos adquiridos por la universidad, con la finalidad de apoyar los procesos de la academia, la investigación y la extensión, que responda a las demandas que los avances tecnológicos exigen especialmente en las relacionadas con la comunicación y acceso a la información.

Una alternativa para el logro de los anteriores propósitos lo han liderado diferentes organizaciones, organismos, universidades y asociaciones a nivel internacional como la Organización de las Naciones Unidas para la Educación, la Ciencia y la Cultura (UNESCO); Council of Australian University Librarians (CAUL); International Federation Librarian Association (IFLA); The Society of Collegue, National and University Libraries (SCONUL); American Library Association (ALA); Association of College \& Research Libraries (ACRL), Re Red de Bibliotecas universitarias de España (REBIUN) a través de documentos modelos, informes y normas que fomentan la aplicación la Alfabetización Informacional al identificar "la necesidad de que los ciudadanos/usuarios adquieran competencias en información y resaltan el papel que las bibliotecas desempeñan como facilitadoras de las herramientas necesarias". [6]

En un porcentaje significativo de universidades que implementan programas de formación de usuarios con la metodología de ALFIN, evidencia la ruptura del paradigma de la biblioteca tradicional que está enfocada al desarrollo de colecciones y de préstamo de libros, hacia una biblioteca reconocida como una organización de enseñanza-aprendizaje, más dinámica y formativa, como Centro de Recursos para el Aprendizaje y la Investigación (CRAI), favorecedora del crecimiento de la producción académica e intelectual de la comunidad educativa.

Este tipo de formación es reconocida en el medio académico con los acrónimos: ALFIN [7], [8] o DHI [9]; que surgen del proceso y prácticas que se han dado en contextos educativos y bibliotecarios donde los participantes, adquieren destrezas necesarias para localizar, evaluar y utilizar eficazmente la información en su vida profesional y personal. [10] La Biblioteca General de la Pontifica Universidad Javeriana de Cali en el marco de la formación de usuarios asume el acrónimo de Lau [7], Desarrollo de Habilidades Informacionales $(\mathrm{DHI})$, que coincide con la concepción de competencias genéricas o transversales planteadas en los proyectos institucionales.

\section{FORMACIÓN DE USUARIOS DE LA BIBLIOTECA GENERAL EN LA PONTIFICIA UNIVERSIDAD JAVERIANA}

El concepto de formación de usuarios en la educación superior ha sido mencionada por autores como: Gazpio y Álvarez [11]; Monfasani y Curzel [12], y Somoza-Fernández [13]. La UNESCO en coincidencia con este concepto también propone la siguiente definición: "todo proyecto o programa destinado a orientar e instruir a los usuarios actuales y potenciales, individual o colectivamente, con el objetivo de facilitar: a) El reconocimiento de sus propias necesidades de información; b) La formulación de estas necesidades; c) La utilización efectiva y eficaz de los servicios de información; d) La evaluación de estos servicios". [14]

Partiendo del anterior contexto la Biblioteca General de la Javeriana - Cali en el 2004 comienza a involucrarse en un estudio más profundo de la literatura de formación de usuarios y alfabetización informacional como respuesta a las demandas institucionales que la Sociedad 
de la Información empieza a plantear a la universidad: 1) Incrementar estadísticas en el uso de los recursos documentales, especialmente las bases de datos; 2) Necesidades concretas de usuarios, especialmente docentes en el conocimiento de las tecnologías de información y de los recursos que la biblioteca venía adquiriendo; 3) Crecimiento de los grupos de investigación, los cuales demandaban mayor información y acceso a recursos documentales más especializados; 4) Necesidad de conformar un equipo de colaboradores idóneo y con el perfil para asumir los cursos y las inducciones.

Iniciativas de formación a usuarios por parte de la biblioteca; se remonta a la década de los años 90 donde la formación se enfocaba fundamentalmente en inducciones y talleres de bases de datos. Posterior a ello, se formuló la posibilidad de replantear los contenidos, el orden de los mismos y hacer la diferenciación entre lo que se consideraba inducción y bases de datos.

Posteriormente, experiencias de formación como el Curso Desarrollo de habilidades informativas en los usuarios de información de la Universidad de Antioquia [15], [16]; el curso de monitores coordinado por la Vicerrectoría del medio universitario; el programa de inducciones a Neojaverianos; y el taller UNESCO de Alfabetización Informacional "Formando a los formadores", fueron experiencias que impulsaron la aplicación de la metodología sobre competencias informacionales y que llevaron a la formulación y puesta en marcha de iniciativas de formación de usuarios con uso de tecnologías como: juego en el software Clic 3.0 y ambiente flash; un Tour virtual de la Biblioteca en $360^{\circ}$.

A partir del 2009 a raíz de experiencias de formación de carácter innovador como las descritas en el párrafo anterior se llevó a cabo la conformación del equipo de trabajo, para el análisis, planeación estructuración y diseño de un curso virtual de formación de usuarios dirigido al fortalecimiento de estrategias de búsqueda y acceso a la información mediante el uso de la plataforma Blackboard Academic.

El curso virtual se ve enmarcado en el programa de formación de usuarios de la biblioteca que hace referencia a cualquier esfuerzo tendiente a sensibilizar, orientar y educar a la comunidad universitaria de manera individual o colectiva, en el uso eficaz de todos los servicios y recursos de información que ofrece la biblioteca, apoyándose en las potencialidades para el aprendizaje que las características de un entorno simbólico basado en las TIC posee. [17]

Curso virtual de biblioteca como estrategia de formación de usuarios: un viaje por el mundo del conocimiento.

El Curso Virtual como estrategia de formación de usuarios busca garantizar el aprendizaje de las herramientas y el uso de los recursos documentales con los que cuenta la Biblioteca para el apoyo de los procesos de investigación y estudio de los estudiantes, docentes, investigadores y colaboradores de la Universidad a la luz de los elementos teóricos y conceptuales de DHI. El curso consta de 4 módulos como se aprecia en la Figura 1. Para conocer los contenidos, objetivos y destinatarios de cada uno de los módulos.

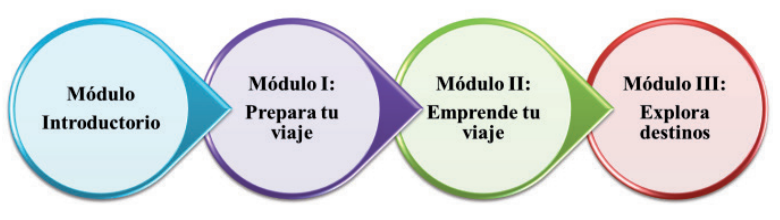

Figura 1. Estructura del Curso

\section{EXPERIENCIA PILOTO: INTEGRACION DEL CURSO VIRTUAL DE BIBLIOTECA A CURSOS PRESENCIALES}

Los objetivos definidos para el estudio piloto fueron: 


\section{General}

Identificar los cambios en el acceso y uso de la información en un grupo de estudiantes de pregrado a partir de la implementación de los módulos del curso virtual de la biblioteca de la Pontificia Universidad Javeriana de Cali.

\section{Especificos}

Implementar los módulos de Biblioteca "Imagina tu viaje" (módulo introductorio), "Prepara tu viaje", "Emprende tu viaje" y "Explora destinos" en 3 cursos de pregrado.

Realizar una evaluación pre-post que permita identificar conocimientos previos y cambios posteriores a la intervención.

\section{Población}

Estudiantes matriculados en los cursos presenciales Expresión oral y escrita y Fundamentos de investigación.

\section{Muestra}

65 Estudiantes de los cursos presenciales Expresión oral y escrita y Fundamentos de investigación

\section{Ruta Metodológica}

Para el periodo 2013-2 fue convocado un grupo de docentes que orientaban cursos dirigidos a favorecer el desarrollo de habilidades de lectura y escritura que implicaba estrategias de búsqueda y acceso a información académica en estudiantes de primeros y últimos semestres de diferentes carreras. Tres docentes aceptaron participar en la integración de los módulos del curso virtual Biblioteca a sus asignaturas.

Para la articulación y exploración del impacto de los módulos de Biblioteca en la muestra de estudiantes, se llevó a cabo la propuesta metodológica que se aprecia en la Figura 2:

Momento 0: Alineación de los objetivos de formación de los módulos virtuales de Biblioteca con los modelos de UNESCO y ACRL. Alineación

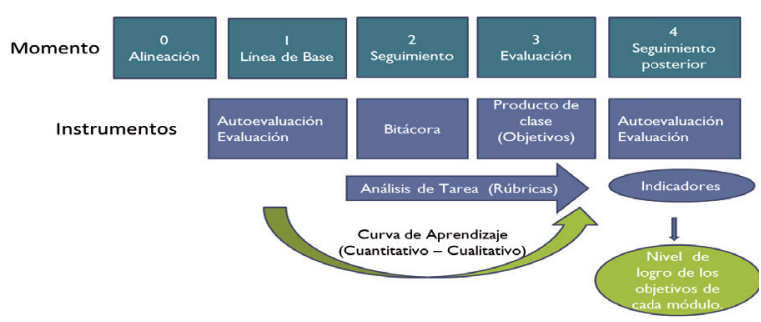

Figura 2. Propuesta Metodológica Pilotaje

de los módulos virtuales de Biblioteca con los objetivos de cada asignatura presencial; Momento 1: Evaluación diagnostica de los estudiantes de cada grupo con base en los objetivos de formación propuestos en el curso virtual (línea de base); Momento 2: Valoración de los desempeños de los grupos en las bitácoras propuestas en el curso virtual, con base en los objetivos del módulo visto; Momento 3: Revisión del producto final de clase que implicaba la aplicación de las habilidades informacionales adquiridas durante el proceso de formación; Momento 4: Evaluación final con base en información inicial a manera de seguimiento al aprendizaje.

Los resultados de los diferentes momentos fueron analizados a partir de las categorías que se describen en la Tabla I.

\section{RESULTADOS DE LA EXPERIENCIA PILOTO}

\section{A. Datos Sociodemográficos.}

La figura 3 presenta los datos sociodemográficos relacionados con el porcentaje de estudiantes de cada curso y la distribución por géneros.

Como se puede evidenciar, el grupo con mayor cantidad de estudiantes es el que se encontraba matriculado en la asignatura Fundamentos de Investigación, el cual representa un $42 \%$ de la población. Así mismo el 68\% de la población de estudiantes pertenece al género masculino.

B. Cambios en el acceso y uso de la información Las figuras 4, 5 y 6 muestran los datos pre (en verde) y post (en azul) alrededor del conocimiento 
Tabla 1. Categorías y Criterios de Análisis

\begin{tabular}{|c|c|c|c|}
\hline Categoría de Análisis & \multicolumn{2}{|l|}{ Criterio de Análisis } & $\begin{array}{l}\text { Técnicas de recolección de } \\
\text { datos }\end{array}$ \\
\hline \multirow[t]{2}{*}{ Datos Sociodemográficos } & \multicolumn{2}{|c|}{$\begin{array}{l}\text { Caracterizar Población por curso (Edad, genero, } \\
\text { carrera, semestre, colegio) }\end{array}$} & Encuesta \\
\hline & \multicolumn{2}{|c|}{ Clasificación de estudiantes por colegios: } & Estadística Descriptiva \\
\hline \multirow[t]{3}{*}{$\begin{array}{l}\text { Apropiación del Curso } \\
\text { Virtual }\end{array}$} & \multicolumn{2}{|c|}{$\begin{array}{l}\text { Pertinencia: Lo adecuado y oportuno del recurso } \\
\text { para su formación }\end{array}$} & \multirow{3}{*}{$\begin{array}{l}\text { Encuesta Modulo Introductorio } \\
\text { (aporte conocimiento) } \\
\text { Grupo Focal } \\
\text { Entrevista } \\
\text { Encuesta Post }\end{array}$} \\
\hline & \multicolumn{2}{|c|}{$\begin{array}{l}\text { Utilidad: Aplicabilidady beneficio del recurso } \\
\text { para su formación }\end{array}$} & \\
\hline & \multicolumn{2}{|c|}{ Sugerencias y Comentarios } & \\
\hline \multirow{2}{*}{$\begin{array}{l}\text { Cambios en el acceso y } \\
\text { uso de información } \\
\text { (Competencias } \\
\text { Informacionales) }\end{array}$} & Curva de aprendizaje & $\begin{array}{l}\text { Contraste resultados de } \\
\text { conocimiento y } \\
\text { adquisición }\end{array}$ & \multirow{2}{*}{$\begin{array}{l}\text { Encuesta } \\
\text { Rubrica Bitácora } \\
\text { Rubrica Productos de clase: } \\
\text { - } \quad \text { Ficha bibliográfica } \\
\text { - } \quad \text { Reseña bibliográfica } \\
\text { - } \quad \text { Anteproyecto de grado }\end{array}$} \\
\hline & $\begin{array}{l}\text { Aumento uso de } \\
\text { servicios de } \\
\text { Biblioteca }\end{array}$ & $\begin{array}{l}\text { Estado Inicial vs Estado } \\
\text { Final }\end{array}$ & \\
\hline
\end{tabular}

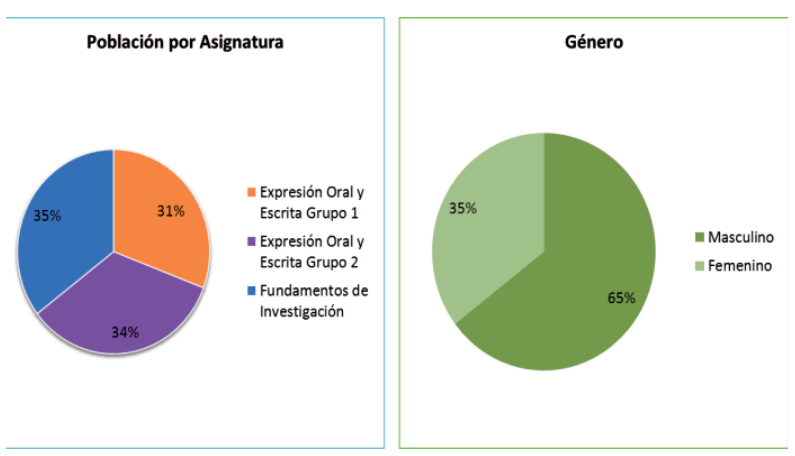

Figura 3. Datos sociodemográficos

y el uso que hacían los estudiantes de los servicios de Biblioteca antes y después del uso de los módulos de Biblioteca en las clases.

Como se evidencia en los gráficos, el conocimiento y uso de los servicios de Biblioteca aumentó después de hacer de uso de los módulos del curso virtual de Biblioteca. En el caso del conocimiento de los servicios de extensión e internos, pasó de un $21 \%$ y $57 \%$ a $86 \%$ y $97 \%$ correspondientemente.

\section{Curva de aprendizaje}

Las figuras 7, 8 y 9 hacen referencia a los cambios derivados del proceso de formación que involucraba a los módulos del curso virtual, representados en una curva de aprendizaje. Estos resultados se relacionan con los objetivos que se buscaban alcanzar en cada módulo y su evidencia de cumplimiento a partir del producto propuesto a su interior (bitácoras) y el producto de clase que también se encontraba alineado a

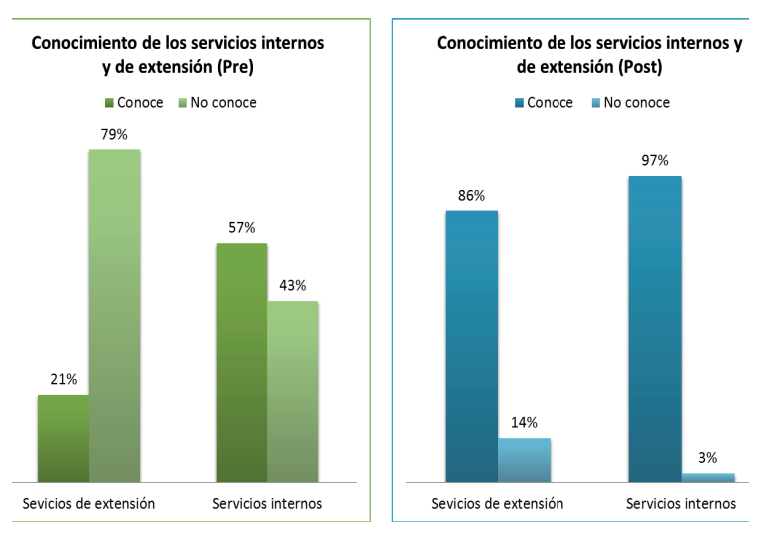

Figura 4. Conocimiento de los servicios de biblioteca
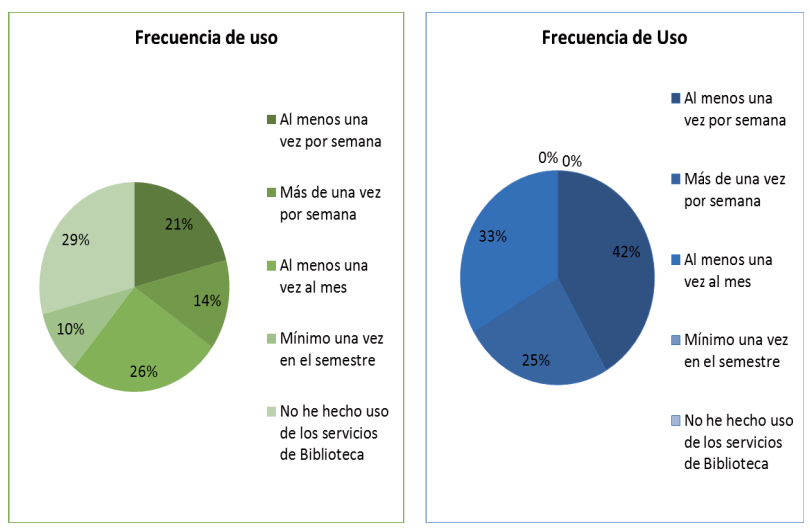

Figura 5. Aumento uso de los servicios de biblioteca
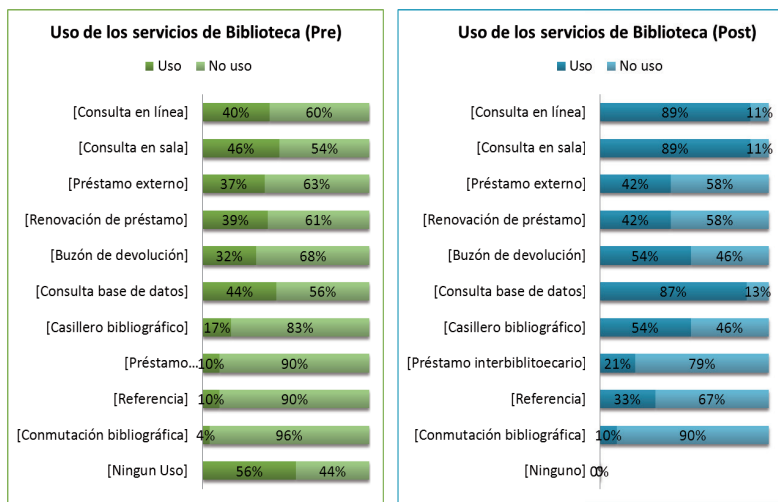

Figura 6. Uso de los servicios de biblioteca

estos objetivos. Los productos fueron calificados a través de rúbricas que permitían ubicar el desempeño de los estudiantes en tres niveles: Básico (1); Medio (2); Alto (3). En los gráficos el eje y corresponde al nivel de desempeño de 1 a 3 y el eje $x$ los objetivos. 


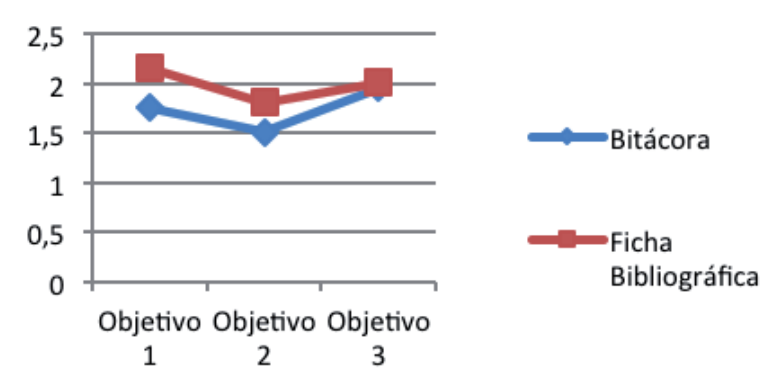

Figura 7. Curva de aprendizaje grupo 1

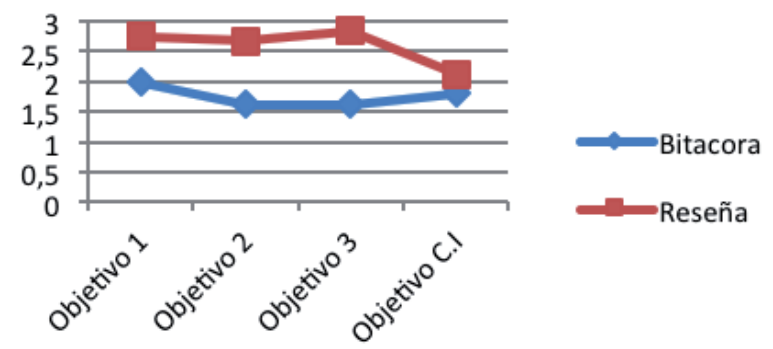

Figura 8. Curva de aprendizaje grupo 2

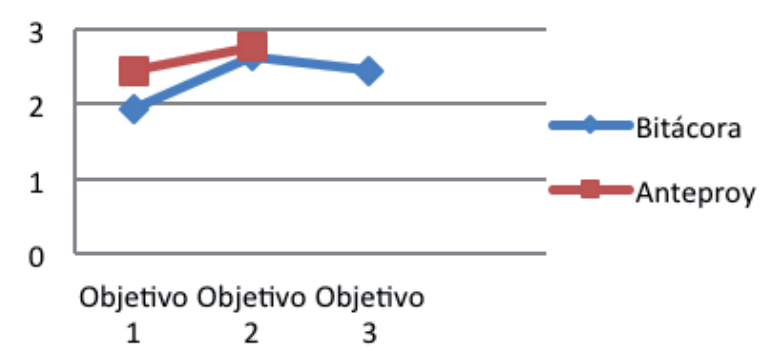

Figura 8. Curva de aprendizaje grupo 3

\section{CONCLUSIONES}

Se identificaron tres ejes fundamentales a los que éste estudio piloto aporta: El papel de la Biblioteca en la formación de usuarios, el uso de la TIC en procesos de enseñanza - Aprendizaje y su relación con el Desarrollo de habilidades informacionales (DHI) y el fortalecimiento de competencias informacionales en estudiantes universitarios.

Con relación al papel de la Biblioteca en la formación de usuarios se evidencia la necesidad de introducir nuevos modelos de aprendizaje donde exista cooperación entre los docentes y líneas de formación transversales del currículo universitario relacionadas con el desarrollo de habilidades para la búsqueda, acceso, uso y evaluación de la información. Como resultado del diseño e implementación del curso virtual con objetivos comunes en las asignaturas hacia la Alfabetización informacional, se demostró la importancia que para docentes, estudiantes y bibliotecólogos debe tener el trabajo colaborativo en el desarrollo de habilidades y competencias de información en un proceso de enseñanza y aprendizaje. En cuanto a la modalidad virtual en la que se desarrolló el curso, se destaca la especial capacidad que ésta tiene para ampliar las posibilidades de aprendizaje gracias a los elementos de carácter estético, perceptual e interactivo que las TIC ofrece y su aporte al desarrollo de diversos contenidos y habilidades relacionadas con el manejo y uso de la información.

Y finalmente la curva de aprendizaje ascendente evidencia el aporte que los módulos del curso virtual y su diseño instruccional tiene para el $\mathrm{DHI}$.

La estrategia de alineación del curso virtual con los objetivos de aprendizaje en las asignaturas del núcleo fundamental y de la línea de formación metodológica seleccionadas para el piloto confirma su aporte.

A partir de los resultados de este estudio piloto se plantearon las siguientes propuestas futuras: Segundo pilotaje con modificaciones metodológicas, segunda experiencia con colaboradores y docentes y una propuesta institucional de MOOC para formación en DHI.

\section{REFERENCIAS}

[1]. Coll, C. "Aprender y Enseñar Con Las TIC: Expectativas, Realidad y Potencialidades." Boletín De La Institución Libre De Enseñanza no. 72 (2008): 17-40.

[2]. UNESCO, ed. Informe Mundial: Hacia Las Sociedades Del Conocimiento, 2005. 
[3]. Villaveces Cardoso, J. L. "Competencia: Cultura Científica, Tecnológica y Manejo De La Información." MEN. http://www.mineducacion.gov.co/1621/ articles-189357_archivo_pdf_cientifica.pdf (accessed Mayo/19, 2014).

[4]. Uribe Tirado, A. and Machett's Penagos, L. "Estado Del Arte De La Alfabetización Informacional En Colombia." IFLA. http:// www.ifla.org/files/assets/informationliteracy/publications/il-report/colombia2010-es.pdf (accessed Mayo/19, 2014).

[5]. Thompson, James and Reg Carr. La Biblioteca Universitaria: Introducción a Su Gestión. Madrid: Fundación Germán Sánchez RuizPérez; Piramide, 1995.

[6]. Hernández, C. J. "Un Plan De Formación En Competencias De Información a Través De Aulas Virtuales: Análisis De Una Experiencia Con Alumnado Universitario." Revista De Universidad y Sociedad Del Conocimiento 7, no. 2 (2010): 50-62, http://www.redalyc. org/pdf/780/78016225015.pdf.

[7]. Gómez-Hernández, José-Antonio. "Las Bibliotecas Universitarias y El Desarrollo De Las Competencias Informacionales En Los Profesores y Los estudiantes 2010, Vol. 7, n. 2, Pp. 37-47. ." Revista De Universidad y Sociedad Del Conocimiento 7, no. 2 (2010): 33-47, http://eprints.rclis. org/18498/2/v7n2-gomez.pdf.

[8]. Bawden,David. "RevisiónDeLosConceptosDe Alfabetización Informacional y Alfabetización Digital ." Anales De Documentación 5, (2002): 361-408, http://revistas.um.es/analesdoc/ article/view/2261/2251 (accessed Mayo 19 de 2014).

[9]. Lau, Jesus. Directrices Sobre Desarrollo De Habilidades Informativas Para El Aprendizaje Permanente. México: IFLA, 2007.
[10]. Gutiérrez Martín, Alfonso. Alfabetización Digital: Algo Más Que Ratones y Teclas. . 2003rd ed. España: Gedisa, 2003

[11]. Martí Lahera, Y. Alfabetización Informacional: Análisis y Gestión, edited by Alfagrama Ediciones. Buenos Aires: Alfagrama Ediciones, 2007.

[12]. Monfasani, R. and M. Curzel. Usuarios De La Información: Formación y Desafíos. Buenos Aires: Alfagra ediciones, 2006.

[13]. Somoza-Fernández, M. Análisis De Los Tutoriales Web Creados Por Bibliotecas Universitarias: Materiales Formativos Para La ALFIN. Berlin: VDM Verlag Dr. Müller, 2009

[14]. Ceretta Soria, María Gladys. "El Vínculo Interactivo Biblioteca Universitaria-Usuario En El Siglo Xxi: Algunas Consideraciones Para Fortalecer Este Relacionamiento." Encontros Bibli: Revista Electrónica De Biblioteconomia e Ciencia Da Informacao 7, no. 13 (2002): 1-12.

[15]. Escuela Interamericana de Bibliotecología. " Curso Desarrollo De Habilidades Informativas En Los Usuarios De Información." Universidad de Antioquia, 7 al 9 de Junio, 2004.

[16]. Naranjo Vélez, Edilma. Desarrollo De Habilidades Informáticas: Una Forma De Animar a Leer. Medellín, edited by Escuela interamericana de Bibliotecología. Medellín: Escuela interamericana de Bibliotecología, 2003.

[17]. Coll, C. "Psicología De La Educación y Prácticas Educativas Mediadas Por Las Tecnologías De La Información y La Comunicación. UnaMirada Constructivista." Revista Electrónica Sinéctica 25, no. agosto-enero (2004): 1-24. 